\title{
Article \\ Supplementing Glycerol to Inoculum Induces Changes in pH, SCFA Profiles, and Microbiota Composition in In-Vitro Batch Fermentation
}

\author{
Qingtao Gao ${ }^{1,+}$, Kai Li ${ }^{1,+}$, Ruqing Zhong ${ }^{1}{ }^{\mathbb{D}}$, Cheng Long ${ }^{2}$, Lei Liu ${ }^{1}$, Liang Chen ${ }^{1, *}$ and Hongfu Zhang ${ }^{1, *}$ \\ 1 The State Key Laboratory of Animal Nutrition, Institute of Animal Sciences, \\ Chinese Academy of Agricultural Sciences, Beijing 100193, China; qingtao_gao@163.com (Q.G.); \\ xndxlk@139.com (K.L.); zhongruqing@caas.cn (R.Z.); liulei02@caas.cn (L.L.) \\ 2 Animal Science and Technology College, Beijing University of Agriculture, Beijing 102206, China; \\ cheng.long@bua.edu.cn \\ * Correspondence: chenliang01@caas.cn (L.C.); zhanghongfu@caas.cn (H.Z.) \\ + These authors contributed equally to this work.
}

check for updates

Citation: Gao, Q.; Li, K.; Zhong, R.; Long, C.; Liu, L.; Chen, L.; Zhang, H. Supplementing Glycerol to Inoculum Induces Changes in $\mathrm{pH}$, SCFA

Profiles, and Microbiota Composition in In-Vitro Batch Fermentation.

Fermentation 2022, 8, 18. https:// doi.org/10.3390/fermentation8010018

Academic Editor: Alessandro Robertiello

Received: 24 November 2021 Accepted: 28 December 2021 Published: 31 December 2021

Publisher's Note: MDPI stays neutral with regard to jurisdictional claims in published maps and institutional affiliations.

Copyright: (C) 2021 by the authors. Licensee MDPI, Basel, Switzerland. This article is an open access article distributed under the terms and conditions of the Creative Commons Attribution (CC BY) license (https:// creativecommons.org/licenses/by/ $4.0 /)$.

\begin{abstract}
Glycerol was generally added to the inoculum as a cryoprotectant. However, it was also a suitable substrate for microbial fermentation, which may produce more SCFAs, thereby decreased $\mathrm{pH}$ of the fermentation broth. This study investigated the effect of supplementing glycerol to inoculum on in vitro fermentation and whether an enhanced buffer capacity of medium could maintain the $\mathrm{pH}$ stability during in vitro batch fermentation, subsequently improving the accuracy of short chain fatty acids (SCFAs) determination, especially propionate. Two ileal digesta were fermented by pig fecal inoculum with or without glycerol (served as anti-frozen inoculum or frozen inoculum) in standard buffer or enhanced buffer solution (served as normal or modified medium). Along with the fermentation, adding glycerol decreased the $\mathrm{pH}$ of fermentation broth $(p<0.05)$. However, modified medium could alleviate the $\mathrm{pH}$ decrement compared with normal medium $(p<0.05)$. The concentration of total propionic acid production was much higher than that of other SCFAs in antifrozen inoculum fermentation at 24 and $36 \mathrm{~h}$, thereby increasing the variation (SD) of net production of propionate. The $\alpha$-diversity analysis showed that adding glycerol decreased Chao1 and Shannon index under normal medium fermentation $(p<0.05)$ compared to modified medium $(p<0.05)$ along with fermentation. PCoA showed that all groups were clustered differently $(p<0.01)$. Adding glycerol improved the relative abundances of Firmicutes, Anaerovibrio, unclassified_f_Selenomonadaceae, and decreased the relative abundance of Proteobacteria $(p<0.05)$. The relative abundances of Firmicutes, such as Lactobacillus, Blautia and Eubacterium_Ruminantium_group in modified medium with frozen inoculum fermentation were higher than $(p<0.05)$ those in normal medium at $36 \mathrm{~h}$ of incubation. These results showed that adding glycerol in inoculum changed the fermentation patterns, regardless of substrate and medium, and suggested fermentation using frozen inoculum with modified medium could maintain stability of $\mathrm{pH}$, improve the accuracy of SCFA determination, as well as maintain a balanced microbial community.
\end{abstract}

Keywords: glycerol; in vitro fermentation; pH; SCFA; microbiota

\section{Introduction}

Dietary fiber (DF) bring benefits to the animals in term of well-being by improving satiety [1,2] and intestinal health by modulating microbiota and metabolites [3,4]. Fiber-rich ingredients contain high amounts of DF resulting in a greater production of short-chain fatty acids (SCFA) [5], which depended on the action of cellulose-utilizing microorganisms colonized in hindgut whereby organization of the enzymes into cellulosomes [6,7]. In turn, recent studies have shown that dietary fiber shapes intestinal microbiota. Zhao et al. [8] 
reported that different sources of dietary fiber differentially influence microbial composition and SCFA concentrations in the intestines of pig model. Therefore, well know the fermentation characteristics of different fiber ingredient is necessary to guide application.

The in vitro fermentation is a widely used method to characterize the fermentation of fiber-rich ingredients by intestinal or fecal microbes of pigs. The main variation in the in vitro fermentation technique could be attributed to the inoculum and its microbial activity [9]. To improve the fecal microbial survival rates during cryopreservation, glycerol was generally added to the inoculum (final concentration of $10 \%$ ) in studies of in vitro fermentation $[10,11]$ served as anti-frozen inoculum. However, glycerol is the main substrates used to produce propionate in fermentation [12], which increased the concentration of propionate and decreased acetate production in vitro [13] and in vivo [14]. Therefore, the glycerol fermentation may obscure the fermentation characteristic of dietary fiber substrates, thereby changing the fermentation profile of microorganisms. Additionally, the medium used forin vitro fermentation was mainly prepared according to a previous study [15], which is a mineral buffer solution. In addition, the buffer capacity of the medium may not enough to maintain the $\mathrm{pH}$ values of fermentation broth along with fermentation due to the accumulation of SCFA. Bai et al. [16] showed that the $\mathrm{pH}$ of fermentation broth was steadily declined when in vitro fermentation using the normal buffer medium. Indeed, similar results were also shown in other in vitro fermentation studies [11,17]. However, since the SCFAs are readily absorbed or metabolized in hindgut fermentation [18], the $\mathrm{pH}$ of colon chyme decreases only slightly [19], because the optimal $\mathrm{pH}$ for microbiota in the hindgut of pigs is around $7[16,20]$. This indicates that sufficient buffer capacity of medium to maintain the stability of the $\mathrm{pH}$ during fermentation is necessary in the future in vitro fermentation study. As for short-chain fatty acid molar ratios in the large intestine of pigs, the production of acetate is generally the highest, makes up $~ 60 \%$ to $75 \%$ of the total SCFAs, followed by propionate and butyrate [21,22], with the molar ratio of acetate, butyrate, and propionate typically being 60:30:10 in the pig intestinal tract [23]. However, in previous in vitro fermentation studies, the molar ratio of propionate was higher than acetate $(39.5 \%$ vs. $35.5 \%$ or $47.8 \%$ vs. $38.5 \%$ for inulin or chicory root fermentation, respectively) [24]. This indicted that the fermentation patterns were affected or changed due to the decreasing $\mathrm{pH}$ values of fermentation broth. Therefore, a suitable environmental $\mathrm{pH}$ is essential for accuracy and repeatability of fermentation, and the optimization of buffer capacity in the fermentation process is critical.

What is mentioned above indicates that some methodological aspects still need to be investigated before the technique can be used in routine. However, many in vitro studies have focused on assess the fermentation property of different types of fiber-rich feedstuffs using the fermentation method, but considerably fewer have focused on the accuracy and repeatability of the method. Additionally, the substrates using in fermentation study mostly were ingredients or hydrolysis residues that were strikingly low in the degree of similarity to ileal digesta [25-27]. Therefore, two ileal digesta derived from growing pigs fed high-fiber content diets were used as substrates. The objective of this study was to explore the effect of supplementing glycerol to inoculum on in vitro fermentation and whether an enhanced buffer capacity of medium could maintain the $\mathrm{pH}$ stability during in vitro batch fermentation, subsequently improving the accuracy of short chain fatty acids (SCFAs) determination.

\section{Materials and Methods}

\subsection{Substrates}

Two ileal digesta from cannulated pigs fed Barley diet and Wheat shorts diet ( 5 pigs per diet) were obtained from a previous study [28], and mixed within diet. The composition of ileal digesta substrates was showed in Table 1. 
Table 1. Chemical composition of feed ingredients (air-dried basis).

\begin{tabular}{ccccccccc}
\hline Item & DM & CP & NDF & ADF & ADL & TDF & IDF & SDF \\
\hline Digesta of Barley diet & 92.67 & 10.70 & 38.58 & 14.23 & 2.55 & 46.64 & 36.93 & 9.71 \\
Digesta of Wheat shorts diet & 93.31 & 8.97 & 36.33 & 11.86 & 3.41 & 45.53 & 37.92 & 7.61 \\
\hline
\end{tabular}

$\mathrm{DM}$, dry matter. $\mathrm{CP}$, crude protein. NDF, neutral detergent fiber. ADF, acid detergent fiber. ADL, acid detergent lignin. TDF, total dietary fiber. IDF, insoluble dietary fiber. SDF, soluble dietary fiber.

\subsection{Preparation of Inoculum and Standardization}

To reduce the variation between animals, the inoculum was prepared by mixing the feces of five pigs. Briefly, five barrows (Duroc $\times$ Landrace $\times$ Large White, bodyweight: $50 \mathrm{~kg}$ approximately) were randomly selected from a same litter as the source of fecal inoculum. Animals were fed with a standard corn-soybean meal and without the use of antibiotics in the last three months before feces collection. The feces were collected directly in plastic bags saturated with $\mathrm{CO}_{2}$. An equal amount of feces was taken from each animal and mixed in a bag and prepared duplicates. One part of mixed feces was diluted 5 times $(\mathrm{m} / \mathrm{v})$ with pre-warmed buffer solution (PBS) and subjected for $60 \mathrm{~s}$ to a mechanical pummeling using a stomacher. The diluted mixture was filtered through four layers of sterile gauze with a $250 \mu \mathrm{m}$-mesh screen and then added to $10 \%$ glycerin $(v / v)$ which served as anti-freezing fecal inoculum, stored at $-80^{\circ} \mathrm{C}$ until further in vitro fermentation. Another mixed feces were directly stored at $-80^{\circ} \mathrm{C}$, waited for preparation to frozen fecal inoculum same to the above description but without adding glycerin. All the processes were operated in an anaerobic operator which was continuously supplied with $\mathrm{CO}_{2}$.

\subsection{Medium Solution}

In this study, two different buffering capacities of the medium were used. The normal medium was prepared as previously describe ( $\mathrm{pH}$ 6.8; Menke and Steingass [15]), which formulated with $8.3 \mathrm{~g} / \mathrm{L} \mathrm{NaHCO}_{3}, 0.95 \mathrm{~g} / \mathrm{L} \mathrm{NH}_{4} \mathrm{HCO}_{3}, 1.35 \mathrm{~g} / \mathrm{L} \mathrm{Na}_{2} \mathrm{HPO}_{4}, 1.47 \mathrm{~g} / \mathrm{L} \mathrm{KH}_{2} \mathrm{PO}_{4}$, $0.14 \mathrm{~g} / \mathrm{L} \mathrm{MgSO} \cdot 7 \mathrm{H}_{2} \mathrm{O}, 0.34 \mathrm{~g} / \mathrm{L} \mathrm{Na}_{2} \mathrm{~S} \cdot 9 \mathrm{H}_{2} \mathrm{O}, 80 \mathrm{mg} / \mathrm{L} \mathrm{NaOH}, 1.6 \mathrm{mg} / \mathrm{L} \mathrm{CaCl} 2 \cdot 2 \mathrm{H}_{2} \mathrm{O}$, $1.2 \mathrm{mg} / \mathrm{L} \mathrm{MnCl}_{2} \cdot 4 \mathrm{H}_{2} \mathrm{O}, 0.12 \mathrm{mg} / \mathrm{L} \mathrm{CoCl}_{2} \cdot 6 \mathrm{H}_{2} \mathrm{O}, 0.96 \mathrm{mg} / \mathrm{L} \mathrm{FeCl} \cdot 6 \mathrm{H}_{2} \mathrm{O}$ and $1.19 \mathrm{mg} / \mathrm{L}$ resazurin. For improving the buffering capacity of medium, the concentration of phosphate buffer was modified to $3.30 \mathrm{~g} / \mathrm{L} \mathrm{Na}_{2} \mathrm{HPO}_{4}, 3.29 \mathrm{~g} / \mathrm{L} \mathrm{KH}_{2} \mathrm{PO}_{4}$ to make the $\mathrm{pH}$ of the buffer equal to 6.8 which served as modified medium. The preparation was carried out under a constant stream of $\mathrm{CO}_{2}$, and subsequently, the two media were bubbled for $30 \mathrm{~min}$ with $\mathrm{CO}_{2}$.

\subsection{Experimental Design and In Vitro Fermentation Procedures}

This experiment was conducted using a completely randomized design. Four treatments including anti-frozen or frozen inoculum fermented in standard or enhanced buffer capacity medium were conducted (Figure 1). Each substrate was added to 21 glass bottles ( 4 for $\mathrm{pH}, \mathrm{SCFA}$ and 3 for microbiota at $12 \mathrm{~h}, 24 \mathrm{~h}$ and $36 \mathrm{~h}$, respectively). Blank glass bottles without substrates were used as controls (4 replicates at each sampling time). Sampling time were based on the hindgut transit time considered to be on average $36 \mathrm{~h}$ (ranging from 24.8 to $48.2 \mathrm{~h}$ ) for growing pigs fed a high fiber diet [29]. Briefly, substrates were exactly weighted at $0.2 \mathrm{~g}$ in $100 \mathrm{~mL}$ glass bottles, blended with $60 \mathrm{~mL}$ sterile medium and $5 \mathrm{~mL}$ inoculum, then the bottles were sealed and incubated at $39^{\circ} \mathrm{C}$ in an air-ventilating, temperature-controlled incubator. All steps were operated in the same condition as the inoculum producing process. 


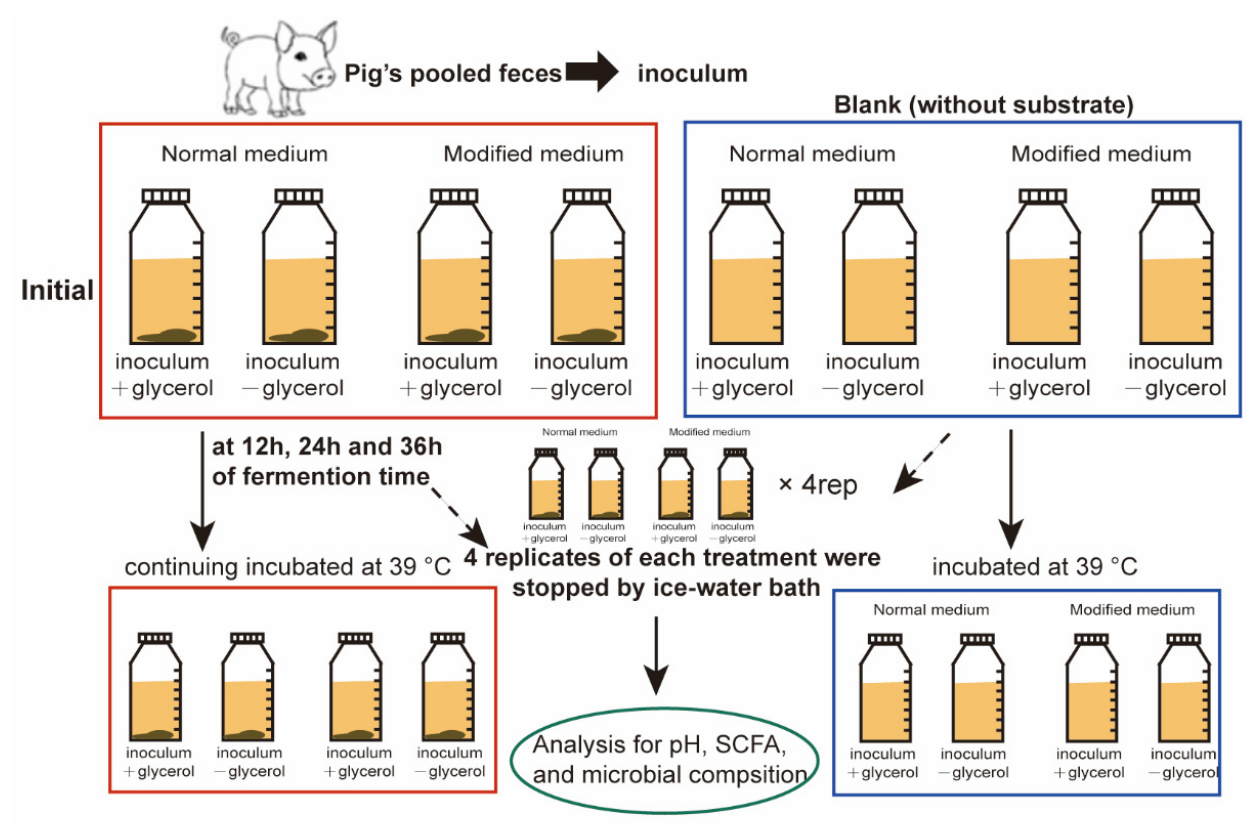

Figure 1. Study design.

At each sampling time, 4 replicates of the fermentation for each treatment were stopped using the ice-water bath. After $\mathrm{pH}$ detection, fermentation broth was centrifuged $\left(10,000 \times g, 10 \mathrm{~min}, 4^{\circ} \mathrm{C}\right)$, the supernatant and the precipitate were stored at $-80{ }^{\circ} \mathrm{C}$ until further analysis.

\subsection{Sample Detection}

\subsubsection{Chemical Analysis}

Digesta were ground to pass through a $0.42-\mathrm{mm}$ sieve and thoroughly mixed before analysis. Samples were analyzed for DM and CP [30]. Total dietary fiber (TDF), SDF and IDF were analyzed according to AOAC procedures with slightly modified [30]. Neutral detergent fiber (NDF), acid detergent fiber (ADF), and acid detergent lignin (ADL) were measured using a modified method [31] with filter bags (Model F57; Ankom Technology, Macedon, NY, USA) and fiber analyzer equipment (ANKOM200 Fiber Analyzer, Ankom Technology).

\subsubsection{Measurement of SCFA Production}

Fermentation supernatants were analyzed for SCFA production (acetate, propionate, butyrate, isobutyrate, valerate, isovalerate) using the method described by the previous study with slight modifications [3]. Briefly, one milliliter of supernatant was mixed with $0.25 \mathrm{~mL} \mathrm{25 \%}(\mathrm{v} / \mathrm{v})$ metaphosphoric acid and kept at room temperature for at least $30 \mathrm{~min}$. Then, the sample was centrifuged at $12,000 \times \mathrm{g}$ for $10 \mathrm{~min}$ at $4{ }^{\circ} \mathrm{C}$ and subjected for analysis on a Gas Chromatograph System (Agilent HP6890 Series, Santa Clara, CA, USA). Branched-chain fatty acids (BCFA) were calculated as the sum of i-butyrate, n-valerate, and i-valerate. SCFAs and BCFA were expressed in mmol/g substrate. Productions of SCFA after fermentation were corrected for the amounts produced in the blank bottles.

\subsubsection{Microbiota Composition Analysis}

Three samples of each treatment for digesta of Barley diet was selected to detect the bacterial community at each time point because the effects of the treatments on the $\mathrm{pH}$ and SCFA production were similar among the two digesta. Centrifugation pellets were extracted for total bacterial genomic DNA using a commercial kit (QIAamp ${ }^{\circledR}$ DNA Stool Mini Kit; QIAGEN, Crawley, UK), following the manufacturer's instructions. For the bacterial 16S ribosomal RNA (rRNA) gene, barcoded amplicons from the V3-V4 region of 16S rRNA 
genes were generated by a 2-step PCR protocol, and the 16S rRNA gene was sequenced on the Illumina HiSeq sequencing platform, as described previously [32]. The clustering of V3-V4 rRNA reads at 97\% nucleotide sequence into operational taxonomic units (OTUs) was performed using UPARSE version 7.1 [33]. OTUs that contained $0.01 \%$ of total reads were filtered out. The Ribosomal Database Project classifier (RDP, version 11.1, http:/ / rdp. cme.msu.edu/, accessed on 30 November 2021) was used for taxonomic assignment [34].

\subsection{Statistical Analysis}

Each bottle was utilized as an experimental unit. One-factor ANOVA and the Duncan multiple comparison method were used to assess group differences in $\mathrm{pH}$, SCFA and $\alpha$ diversity index measurements using SAS 8.0 (SAS Institute, Inc., Cary, NC, USA). Statistical variations were estimated by the standard deviation (SD). The Bray-Curtis similarity principal coordinates analysis were tested for significant separation using the ANOSIM permutational test ( 999 permutations) by Majorbio I-Sanger Cloud Platform (www.i-sanger. com, accessed on 30 November 2021). Statistical significance of the relative abundance at the taxonomic levels among the 4 groups was compared using the non-parametric Kruskal-Wallis $\mathrm{H}$ test. All statistical significance was set at $p<0.05$.

\section{Results}

\subsection{Changes of $p H$ Values in Fermentation Broth during In-Vitro Fermentation}

The $\mathrm{pH}$ changes were investigated among different medium and inoculum treatments in this study (Figure 2). Anti-frozen inoculum (added glycerol) had a significant lower $(p<0.05) \mathrm{pH}$ value of fermentation broth compared to frozen inoculum at $12 \mathrm{~h}$ (Figure 2A,B), $24 \mathrm{~h}$ (Figure 2C,D) and $36 \mathrm{~h}$ (Figure 2E,F) of fermentation for 2 substrates, regardless of media. However, for anti-frozen inoculum fermentation, the $\mathrm{pH}$ of fermentation broth under modified medium was higher than $(p<0.05)$ that under normal medium counterpart. From the perspective of the time effect (Figure 2G,H), anti-frozen inoculum fermentation under normal or modified medium (N_AF or M_AF group) decreased the pH values of fermentation broth alone with fermentation, and compared with N_AF group fermentation, the M_AF group effectively slowed down the decrease of $\mathrm{pH}$ in fermentation broth $(p<0.05)$, irrespective of substrates.

\subsection{SCFAs Profiles during Fermentation among Different Medium and Inoculum Treatments}

The amount of SCFAs production was determined at 12, 24 and $36 \mathrm{~h}$ of fermentation (Figure 3). The acetic acid production in fermentation of two digesta substrates among the four groups was significantly different at all time points $(p<0.05)$. For frozen inoculum fermentation, modified medium (M_F group) produced less acetate than normal medium (N_F group) at $12 \mathrm{~h}$ of fermentation, but more acetate was produced at $36 \mathrm{~h}$ of fermentation $(p<0.05$; Figure 3A,B); for anti-frozen inoculum fermentation, modified medium (M_AF group) produced the highest acetate, which was lowest in normal medium (N_AF group) during all fermentation time points $(p<0.05$; Figure $3 \mathrm{~A}, \mathrm{~B})$. The concentrations of propionate were greater in normal medium (N_F group) than modified medium (M_F group) under frozen inoculum fermentation at $12 \mathrm{~h}(p<0.05$; Figure 3D), but there was no significant difference between N_F group and M_F group at $24 \mathrm{~h}$ and $36 \mathrm{~h}(p>0.05$; Figure 3D) during fermentation of barley diet digesta. Moreover, there was no significant difference for propionate production between N_F group and M_F group at all sampling times during fermentation of wheat shorts diet digesta $(p>0.05$; Figure $3 C)$; under the anti-frozen inoculum fermentation, normal medium (N_AF group) produced less propionate than modified medium (M_AF group) during fermentation of two digesta with the exception of the barley digesta fermentation at $24 \mathrm{~h}(p<0.05$; Figure 3C,D). Under frozen inoculum fermentation, modified medium (M_F group) had a lower butyrate and BCFA production than normal medium (N_F group) at $12 \mathrm{~h}(p<0.05$; Figure 3E-H), but had no longer significant difference at $36 \mathrm{~h}(p>0.05$; Figure $2 \mathrm{E}-\mathrm{H})$ during fermentation of two digesta. Nevertheless, under anti-frozen inoculum fermentation, modified medium (M_AF group) 
also had a lower butyrate and BCFA production than that in normal medium (N_AF group) both for digesta of barley diet and wheat shorts fermented $(p<0.05$; Figure $3 \mathrm{E}-\mathrm{H})$.

A

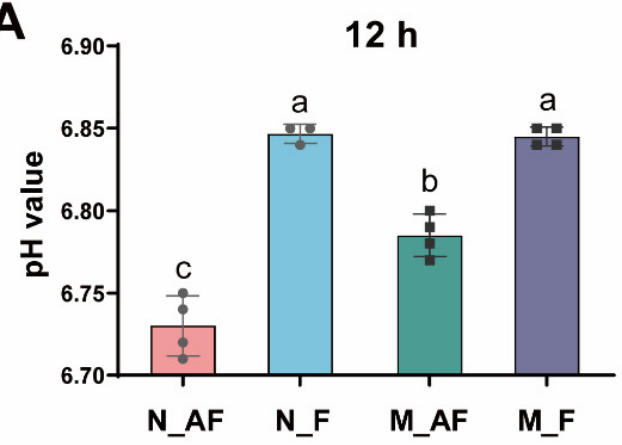

C

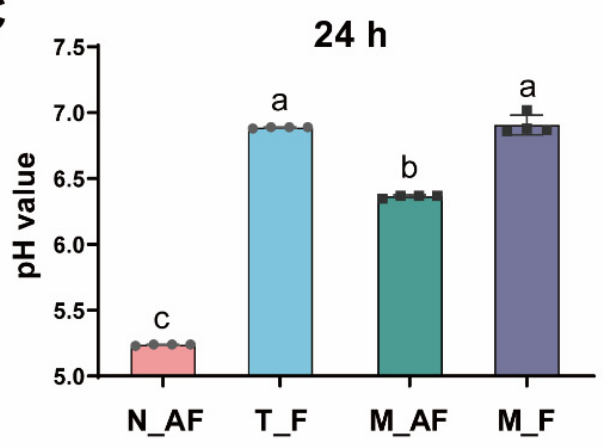

E

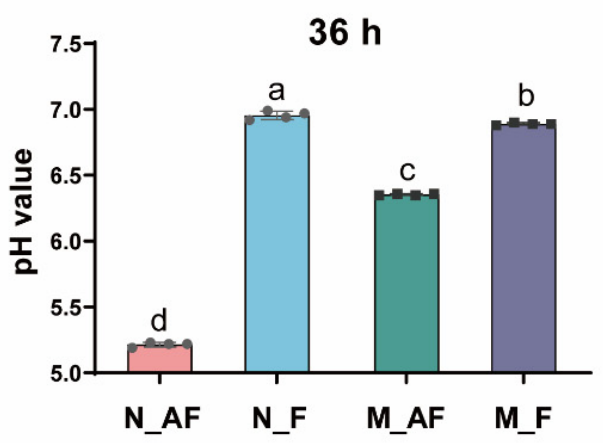

G

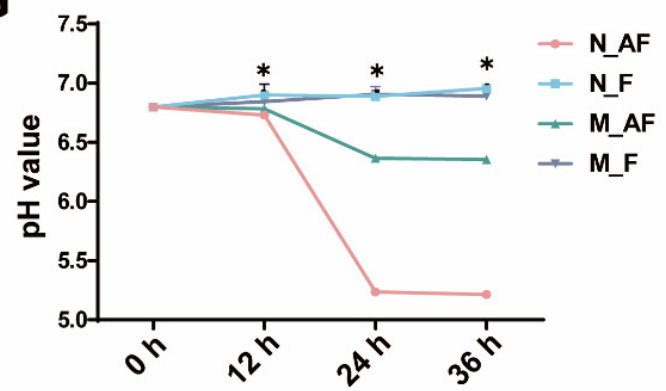

B

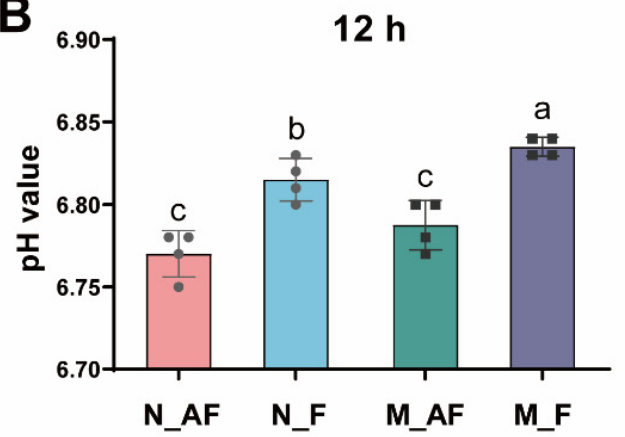

D

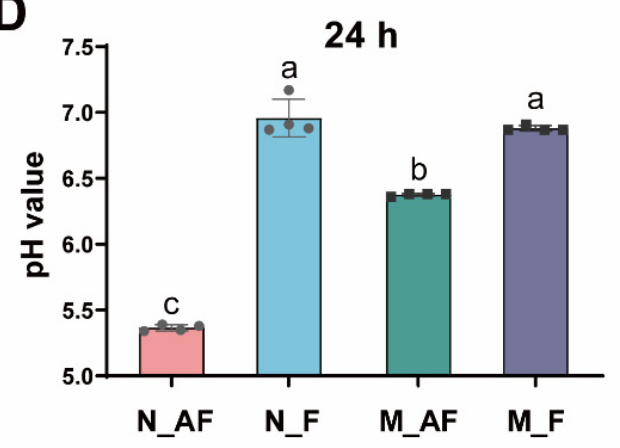

$\mathbf{F}$

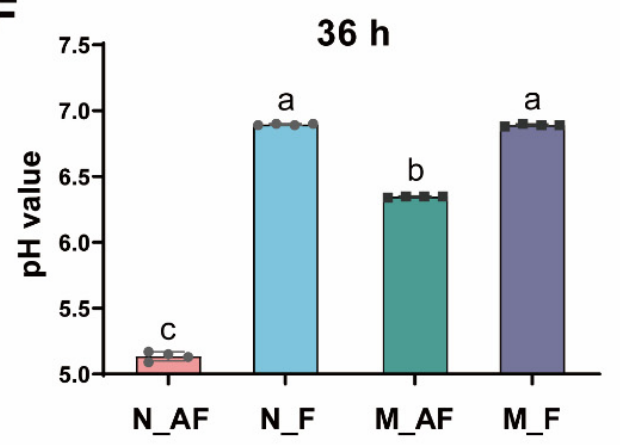

H

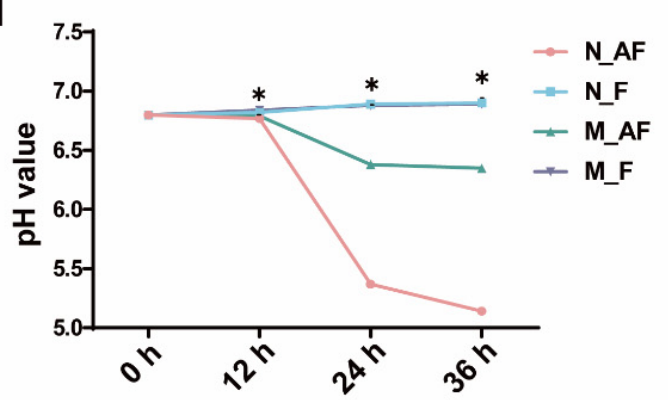

Figure 2. Effect of different treatments on $\mathrm{pH}$ of fermentation broth for digesta of Barley diet (A,C,E) and digesta of wheat shorts diet $(\mathbf{B}, \mathbf{D}, \mathbf{F})$ at each sampling time and $\mathrm{pH}$ changes for digesta of Barley $\operatorname{diet}(\mathbf{G})$ and digesta of wheat shorts $\operatorname{diet}(\mathbf{H})$ over time during fermentation. The results presented as the mean values \pm SEM. $\left(n=4\right.$; Different letters mean $p<0.05 ;{ }^{*}$ mean $p<0.05 . \mathrm{N} \_\mathrm{AF}$, normal medium with anti-frozen inoculum; N_F, normal medium with frozen inoculum; M_AF, modified medium with anti-frozen inoculum; M_F, modified medium with frozen inoculum). 
A
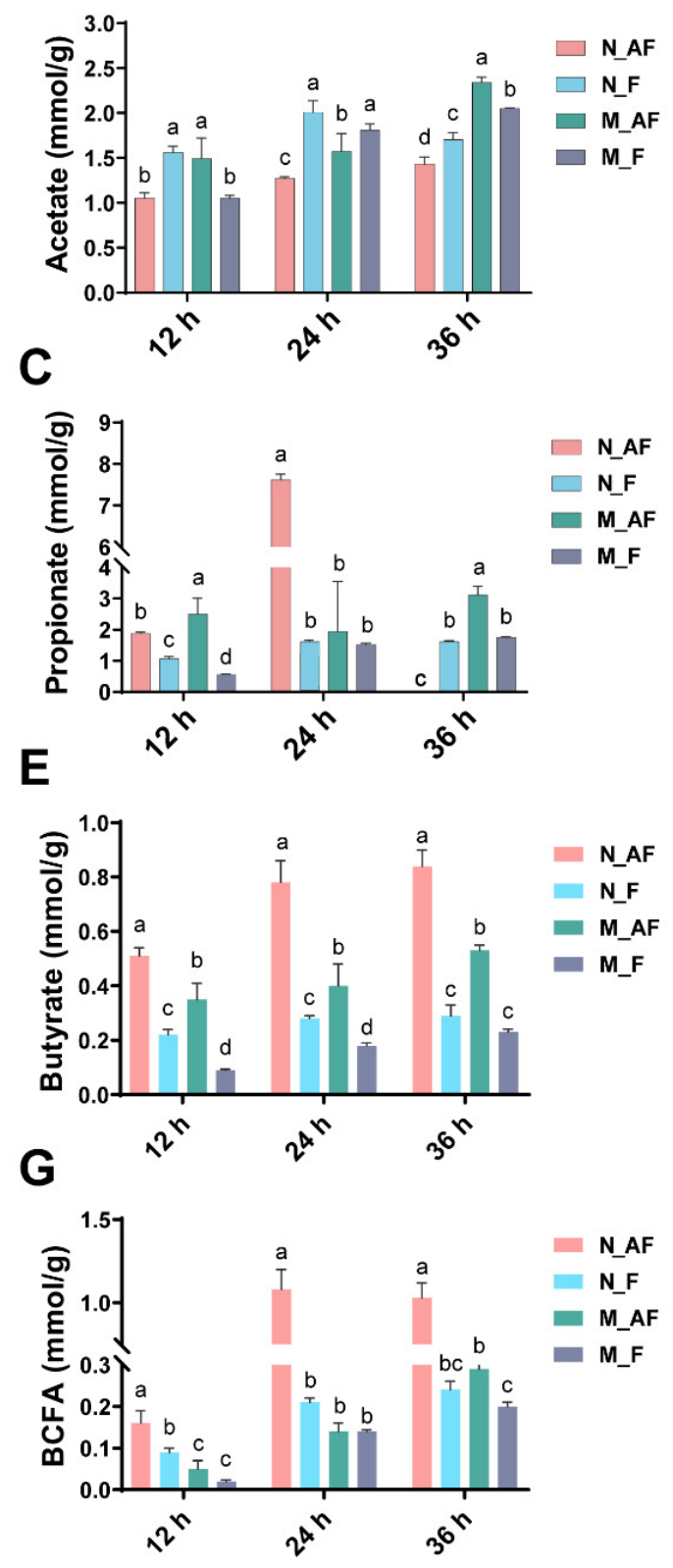

B
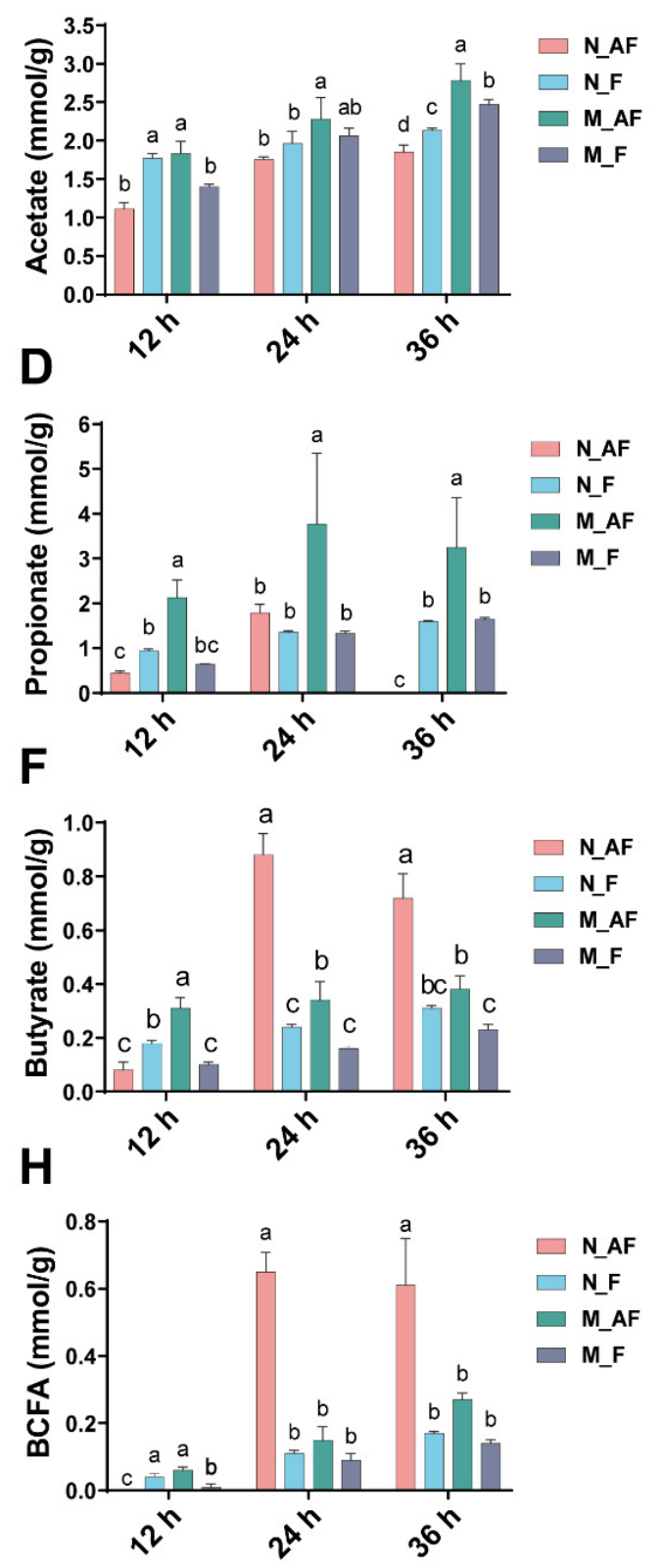

Figure 3. Changes of SCFAs production in the fermentation broth for digesta of Barley diet (A,C,E,G) and wheat shorts diet $(\mathbf{B}, \mathbf{D}, \mathbf{F}, \mathbf{H})$ at each sampling time during fermentation. The values were corrected for the amounts produced in the blank bottles and presented as the mean values. ( $n=4$; Different letters mean $p<0.05$; BCFAs were calculated as the sum of i-butyrate, $n$-valerate, and $\mathrm{i}$-valerate).

From the results of control groups (without substrates) at $24 \mathrm{~h}$ and $36 \mathrm{~h}$ during fermentation among different treatments (Figure 4), the concentration of propionic acid was much higher than that of other SCFA in the anti-frozen inoculum (N_AF or M_AF groups). Additionally, frozen inoculum with modified medium (M_F group) fermentation produced lower SCFA than other treatment groups. 
$12 \mathrm{~h}$

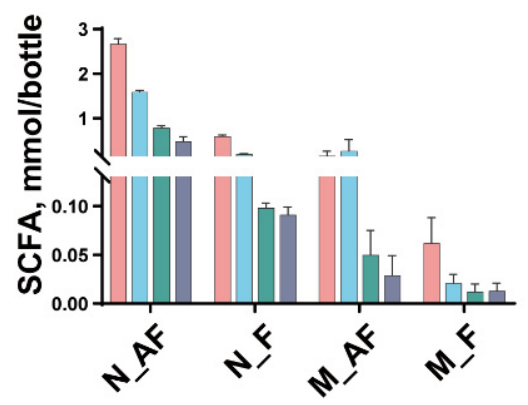

$24 \mathrm{~h}$

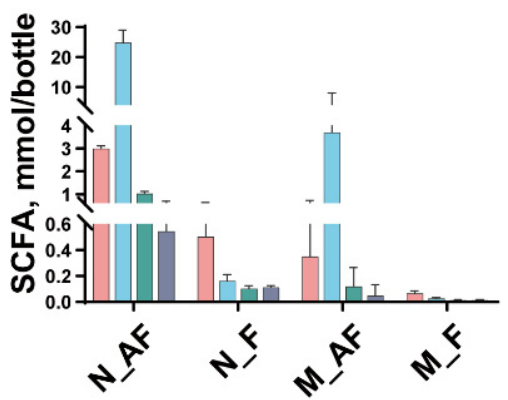

$36 \mathrm{~h}$

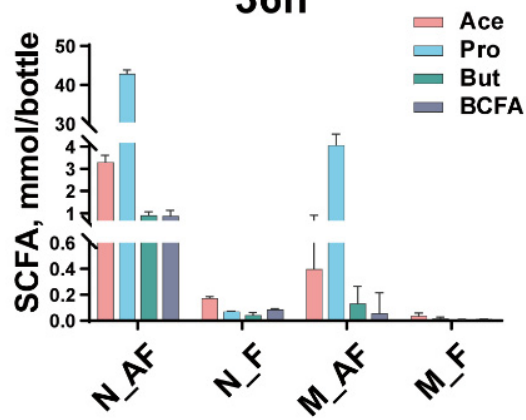

Figure 4. The amount of SCFAs in the fermentation broth for control (without substrate) among different treatments at each sampling time during fermentation.

\subsection{The Changes in the Microbial Composition during Fermentation}

The difference in alpha-diversity of microbiota in fermentation among different treatments was presented by Chao 1 and Shannon indexes (Figure 5A,B). At $12 \mathrm{~h}$ of fermentation, frozen inoculum decreased Chao 1 index both in normal and modified medium and decreased Shannon index in normal medium fermentation ( $p<0.05$; Figure $5 \mathrm{~A}, \mathrm{~B})$. At $24 \mathrm{~h}$ of fermentation, modified medium with anti-frozen inoculum (M_AF group) had the highest Chao 1 and Shannon indexes among four treatments $(p<0.05$; Figure $5 \mathrm{~A}, \mathrm{~B})$. However, at $36 \mathrm{~h}$, there was no significant difference for Chao 1 and Shannon indexes between frozen and anti-frozen inoculum under modified medium fermentation $(p>0.05$; Figure $5 \mathrm{~A}, \mathrm{~B})$. Moreover, principal coordinate analysis ( $\mathrm{PCOA}$ ) based on Bray-Curtis distance showed clear segregation of microbial communities among different treatments at 12, 24 and $36 \mathrm{~h}$ (Figure 5C-E) during fermentation.

A

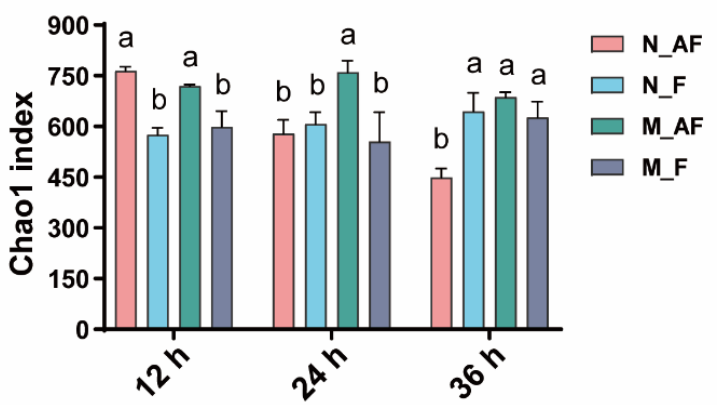

B

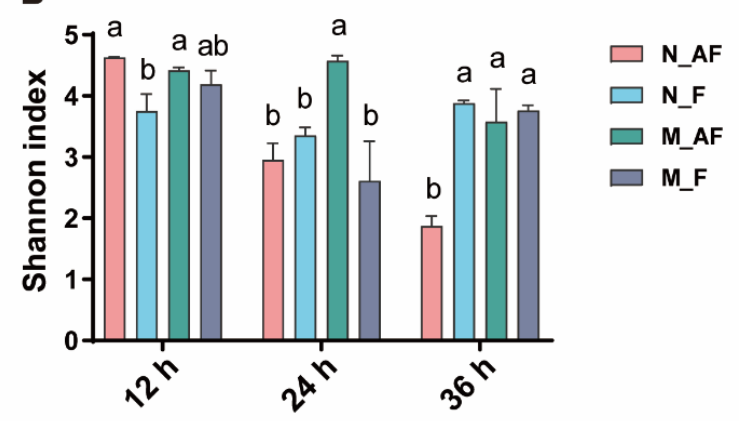

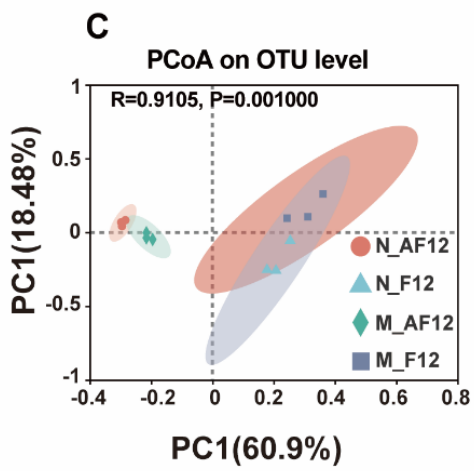

D

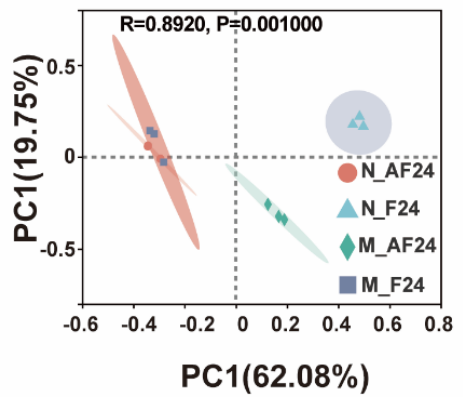

E

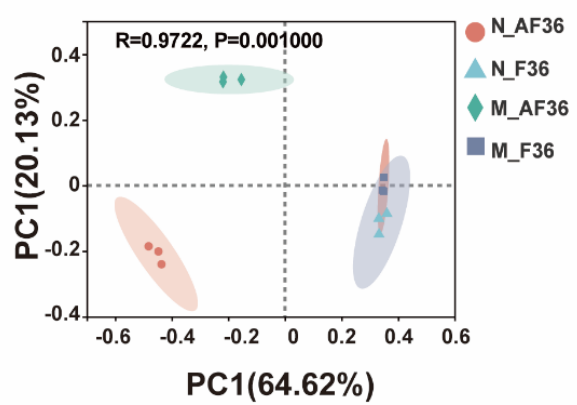

Figure 5. Bacterial diversity during fermentation. Chao1 (A) and Shannon (B) indexes in the

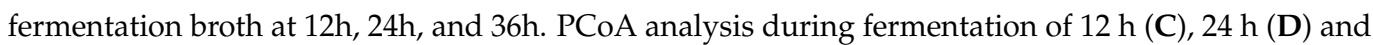
$36 \mathrm{~h}(\mathrm{E})$. Data are represented as mean $(n=3$; Different letters mean $p<0.05)$. N_AF, normal medium with anti-frozen inoculum; N_F, normal medium with frozen inoculum; M_AF, modified medium with anti-frozen inoculum; M_F, modified medium with frozen inoculum. 
To further determine the changes in gut bacterial communities, the bacterial communities at the phylum (Figure 6A) and genus levels (Figure 6B) were analyzed. The results showed Firmicutes and Bacteroidetes were the dominant bacteria among all groups followed by Actinobacteria and Proteobacteria, accounting for almost $97 \%$ of the total sequences. Differences in the relative abundance in fermentation broths among the four treatments at each sampling were further identified using the multiple comparison analysis.

A

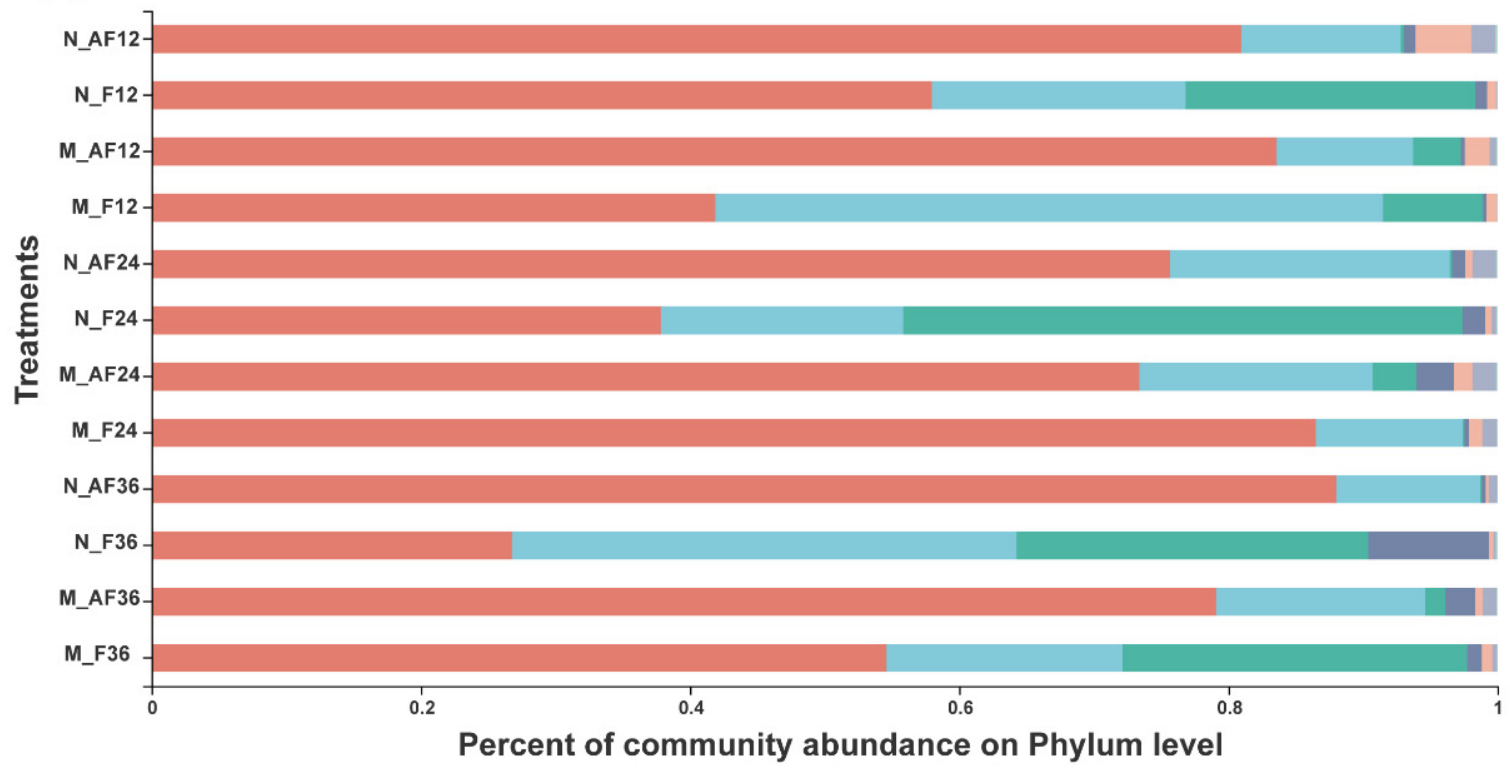

n Firmicutes $\square$ Bacteroidota $\square$ Proteobacteria $\square$ Spirochaetota $\llbracket$ Actinobacteriota $\square$ Desulfobacterota $\llbracket$ others

\section{B}

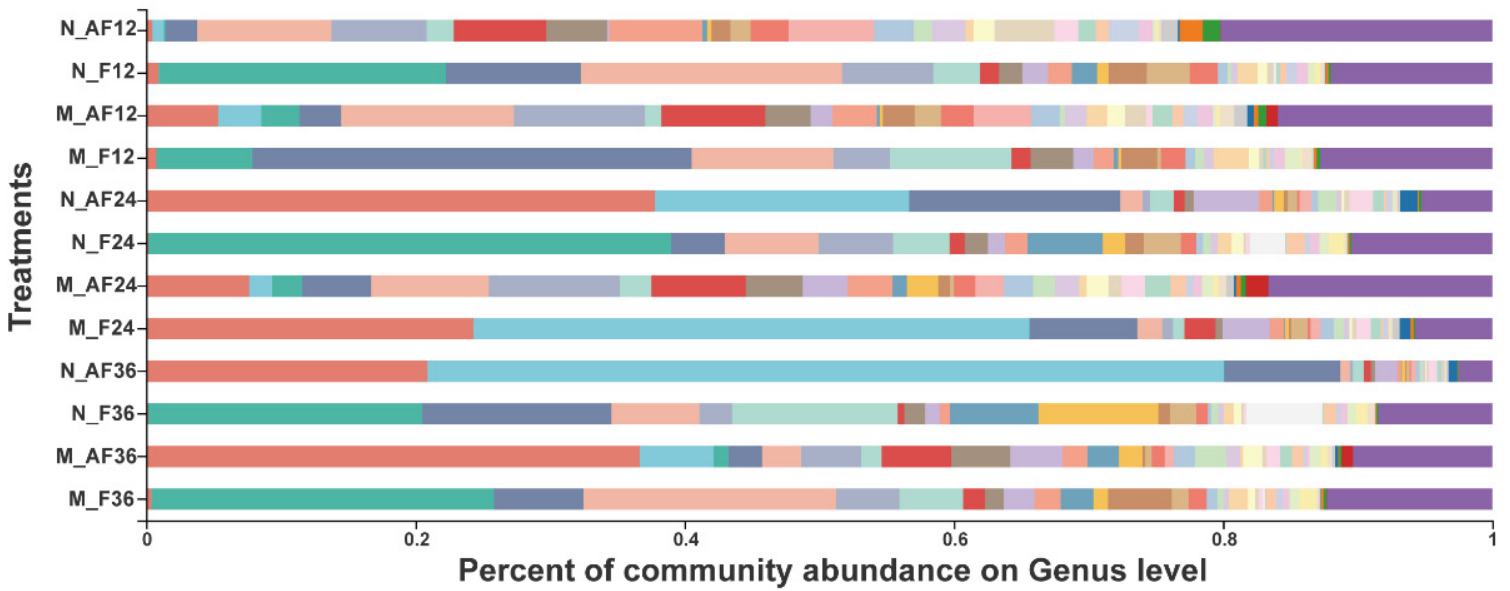

\begin{tabular}{|c|c|c|c|c|c|}
\hline $\begin{array}{l}\text { Anaerovibrio } \\
\text { unclassified_f_Selenomonadacea }\end{array}$ & - Clostridium_sensu_stricto_1 & Megasphaera & $\begin{array}{l}\text { Solobacterium } \\
\text { Parabacteroides }\end{array}$ & $\begin{array}{l}\text { Ruminococcus } \\
\text { Collinsella }\end{array}$ & $\begin{array}{l}\text { Rikenellaceae_RC9_gut_group } \\
\text { unclassified_f_Lachnospiraceae }\end{array}$ \\
\hline Escherichia-Shigella & Phascolarctobacterium & Shuttleworthia & Desulfovibrio & Oribacterium & Eothers \\
\hline Prevotella & Blautia & Terrisporobacter & Pseudomonas & Eysinibacillus & \\
\hline Lactobacillus & Prevotellaceae_UCG-001 & Subdoligranulum & Faecalibacterium & Christensenellac & ceae_R-7_group \\
\hline Prevotellaceae_NK3B31_group & Eubacterium_ruminantium_group & Catenibacterium & Roseburia & unclassified_f__ & Prevotellaceae \\
\hline
\end{tabular}

Figure 6. The microbial composition at the phylum (A) and genus (B) level with an abundance greater than $1 \%$ in fermentation broth at 12,24 and $36 \mathrm{~h}$. ( $n=3 ; \mathrm{N}_{-} \mathrm{AF}$, normal medium with anti-frozen inoculum; N_F, normal medium with frozen inoculum; M_AF, modified medium with anti-frozen inoculum; M_F, modified medium with frozen inoculum).

On the phylum level, at $12 \mathrm{~h}$ of fermentation (Figure 7A-D), adding glycerol improved $(p<0.05)$ the relative abundance of Actinobacteria decreased $(p<0.05)$ the Proteobacteria 
relative abundance compared to frozen inoculum. However, the modified medium (M_A froup) had a higher abundance $(p<0.05)$ of Bacteroidetes than normal medium (N_A group), and had a trend toward decreased Proteobacteria abundance $(p=0.06)$ under frozen inoculum fermentation conditions. At $24 \mathrm{~h}$ of fermentation (Figure 7E-G), modified medium (M_F group) fermentation with frozen inoculum showed greater $(p<0.05)$ Firmicutes abundance than normal medium (N_F group), similar to that relative abundance observed in anti-frozen inoculum fermentation (M_AF or N_AF group). In addition, modified medium (M_F group) decreased $(p<0.05)$ the Proteobacteria population in frozen inoculum fermentation. At $36 \mathrm{~h}$ of fermentation (Figure $7 \mathrm{H}-\mathrm{K}$ ), frozen inoculum fermentation had a higher $(p<0.05)$ Proteobacteria population and a lower $(p<0.05)$ Actinobacteria abundance compared to anti-frozen inoculum. However, under frozen inoculum fermentation conditions, the modified medium (M_A group) improved the relative abundance $(p<0.05)$ of Firmicutes and Actinobacteria than normal medium (N_A group).
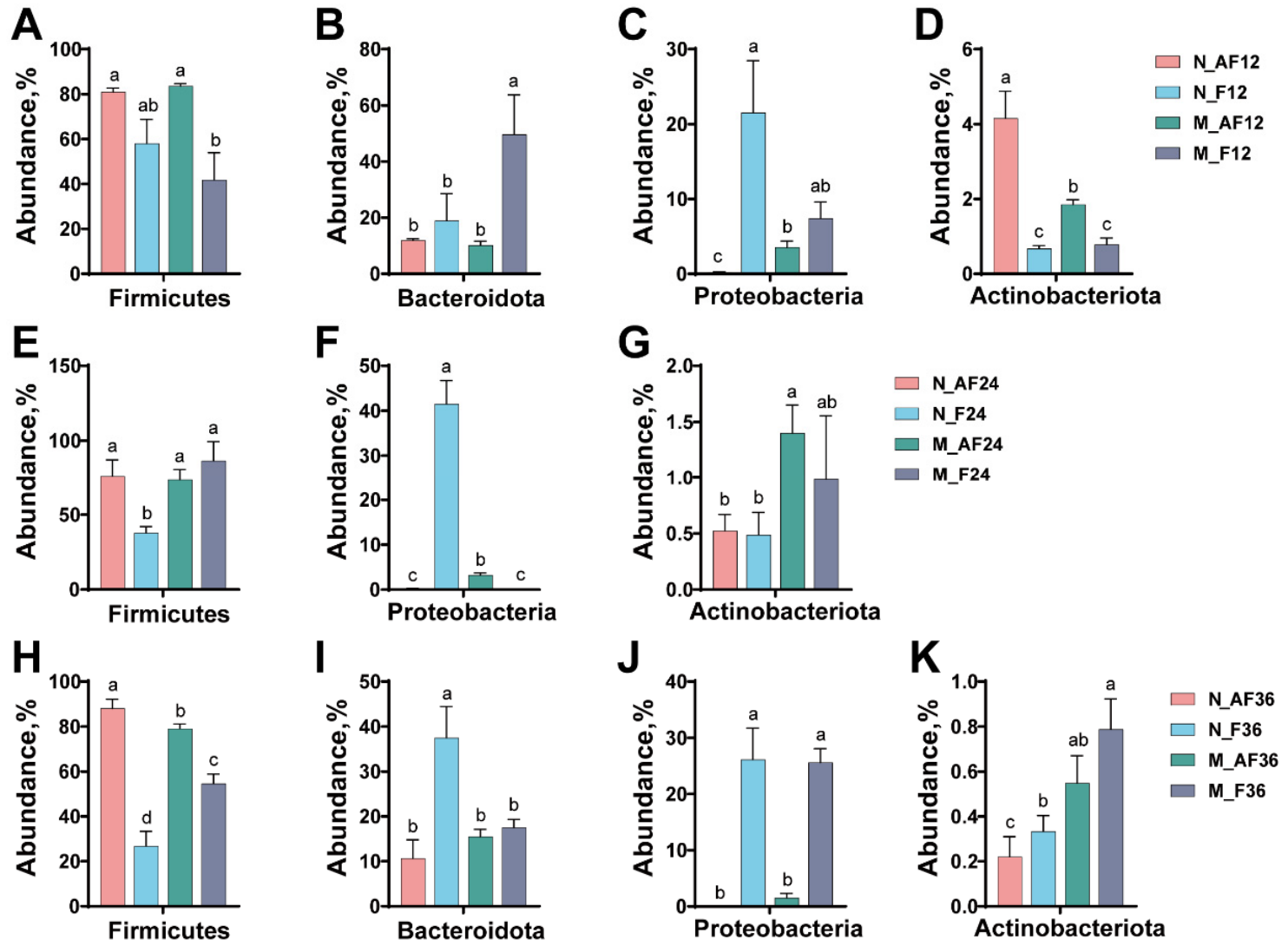

Figure 7. The relative abundance of the predominant bacterial phyla (Firmicutes, Bacteroidetes, Actinobacteria and Proteobacteria) at the time point of $12 \mathrm{~h}(\mathbf{A}-\mathbf{D}), 24 \mathrm{~h}(\mathbf{E}-\mathbf{G})$ and $36 \mathrm{~h}(\mathbf{H}-\mathbf{K})$ during in vitro fermentation using digesta of Barley. Statistical significance was analyzed by Kruskal-Wallis $\mathrm{H}$ test.

At the genus levels, a differential analysis and Welch's Post-hoc test of bacteria among the four treatments at each sampling time was performed. At $12 \mathrm{~h}$ of fermentation (Figure 8A), Escherichia-Shigella, a bacterial to the phylum Proteobacteria, was higher $(p<0.05)$ in the frozen inoculum fermentation (N_F group, M_F group), but modified medium trended to inhibit the relative abundance of Escherichia-Shigella $(p=0.06)$ under the frozen inoculum fermentation. Prevotella belonging to phylum bacteriodetes trended to improve $(p=0.06)$ by modified medium than normal medium under frozen inoculum fermentation. Bacterial genera belonging to Firmicutes, such as Streptococcus, Clostridium_sensu_stricto_1, Blautia, Terrisporobacter, etc., were lower in frozen inoculum (N_F group, M_F group) than in anti-frozen inoculum (N_AF group, M_AF group). At $24 \mathrm{~h}$ of fermentation (Figure 8B), Escherichia-Shigella was the highest in N_F group and the lowest 
in M_F group. The abundance of norank_f_Muribaculaceae, belonging to Bacteroidota, was not different between N_F group and M_F group. As for genera belong to Firmicutes, the abundance of Anaerovibrio and unclassified_f_Selenomonadaceae were higher in M_F group than that in N_F group, the Streptococcus, Clostridium_sensu_stricto_1 and Blautia abundance in M_F group were not different with N_AF group, but lower than that in M_AF group. At $36 \mathrm{~h}$ of fermentation (Figure 8C), Pseudomonas but not Escherichia-Shigella, belonging to a phylum Proteobacteria, was lower in M_F group than that in N_F group. Bacterial genera belonging to phylum Firmicutes, such as Lactobacillus, Clostridium_sensu_stricto_1, Blautia and Eubacterium_ruminantium_group, were improved $(p<0.05)$ in M_A group than N_A group fermentation. As for genera belonging to Bacteroidota, Prevotella, Prevotellaceae_NK3B31_group and Prevotellaceae_UCG-001 were lower in M_F group than N_F group, but higher than M_AF group fermentation.

A
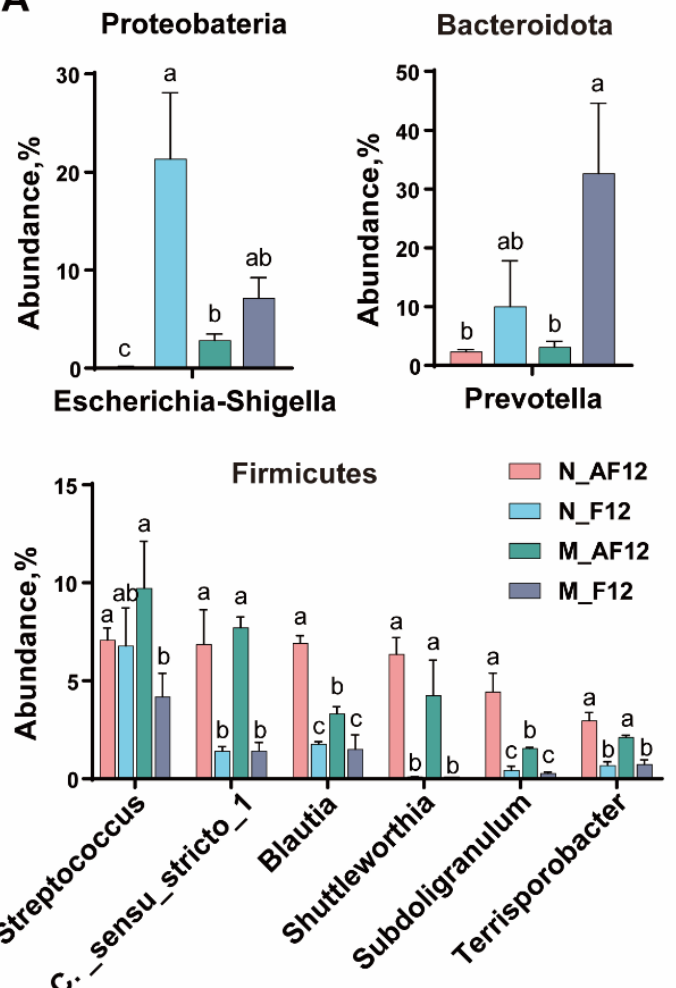

C $-\overline{-}--$
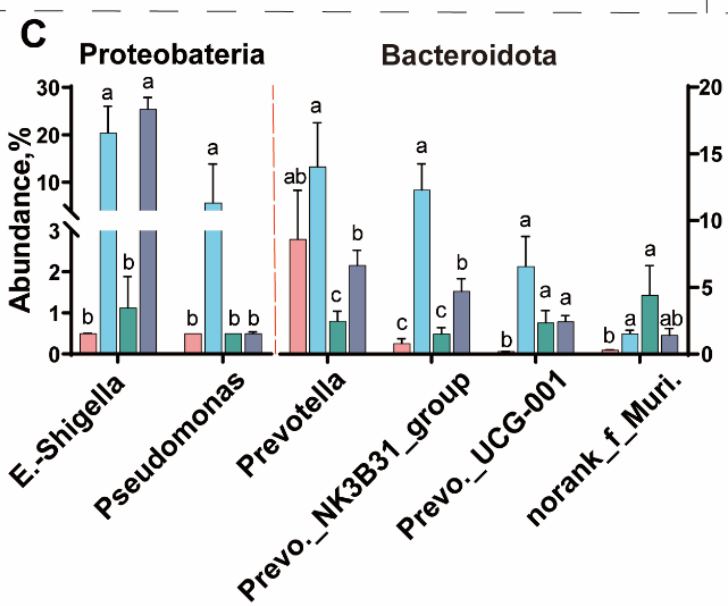

B

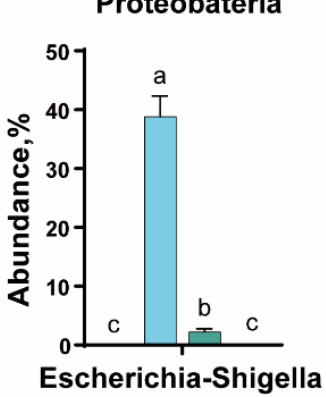

\section{Bacteroidota}

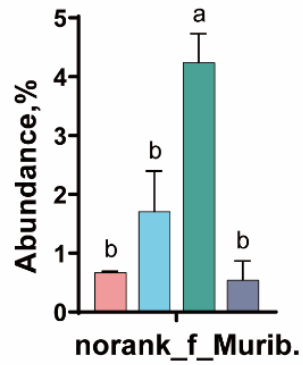

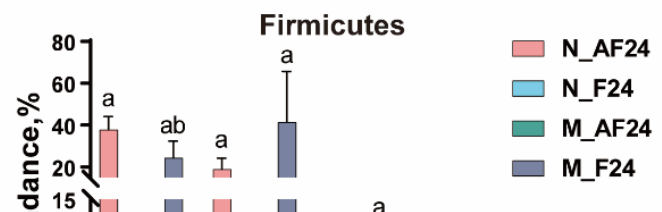
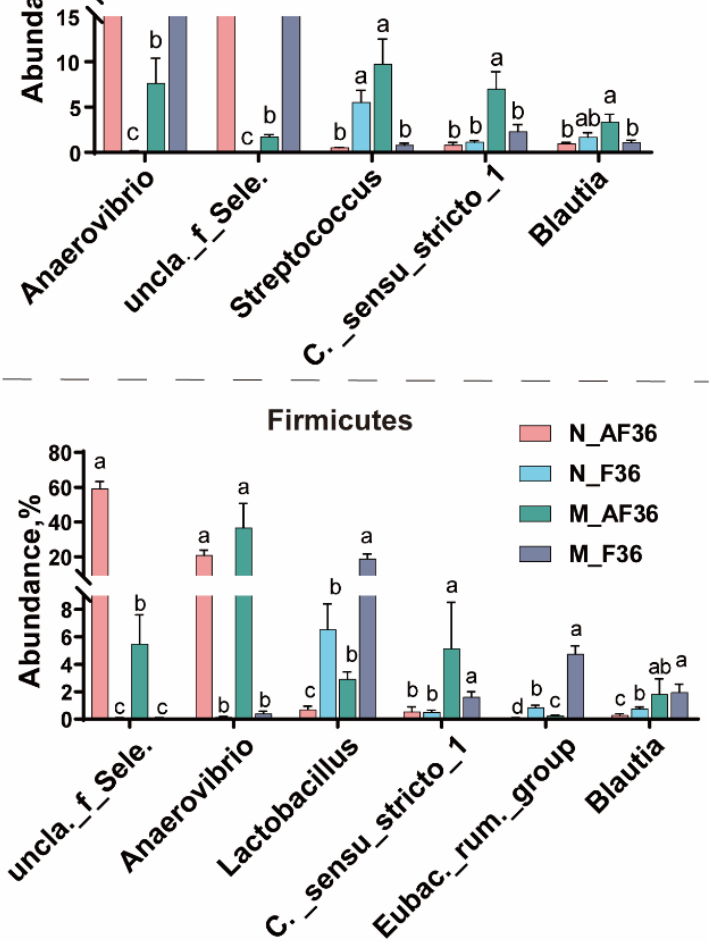

Figure 8. Several differential genera exhibiting the first 15 bacteria among different treatments at $12 \mathrm{~h} \mathrm{(A),} 24 \mathrm{~h}$ (B) and $36 \mathrm{~h}$ (C) during fermentation. Statistical significance was determined by Kruskal-Wallis test. 


\section{Discussion}

A deep understanding of fermentation characteristics of fiber-rich foods or ingredients is helpful for feed selection and utilization. Therefore, it is important to improve the accuracy and repeatability of the assessment method. This research aimed to investigate effects of adding glycerol to inoculum fermented in two media on $\mathrm{pH}$, SCFA production and bacterial community during fermentation, in order to improve the accuracy and repeatability of this method. Comparing the results of $\mathrm{pH}$ changes, SCFA production, and the microbiota under different treatments showed that the inoculum adding glycerol accounted for a large part of the variability.

The buffer solution medium used by Menke and Steingass [15] offered an appropriate environment to the colonic or fecal microflora. In previous study, the original $\mathrm{pH}$ values in the fermentation broth was ranged from 6.7 to 7.0 [25], depending on the inoculum and substrate, which were consistent with $\mathrm{pH}$ values measured in pig large intestines [35]. Along with the fermentation, however, accumulation of SCFA would decrease the $\mathrm{pH}$ values to a different extent that partly depending on the buffer capacity of medium. Therefore, in some in vitro fermentation studies, culture $\mathrm{pH}$ was maintained between 6.7-6.9 using $\mathrm{pH}$ controllers [36]. In the present study, frozen inoculum fermentation under normal or modified medium, the $\mathrm{pH}$ of fermentation broth was ranged from 6.8 to 7 during fermentation fermenting two digesta substrates, which was consistent with the previous report [25]. However, anti-frozen inoculum fermentation under normal medium, the $\mathrm{pH}$ of broth decreased to 5.3 at $24 \mathrm{~h}$ of fermentation, and a further decline was observed at $36 \mathrm{~h}$. Bai et al. [11] found a significantly decreased in $\mathrm{pH}$ values of fermentation broth during in vitro fermentation with $10 \%$ glycerin supplementation, which indicates the buffer capacity of the normal medium was not enough to maintain the $\mathrm{pH}$ values in fermentation process. Expectedly, anti-frozen inoculum fermentation under modified medium, the $\mathrm{pH}$ of fermentation broth decrease is thought to stop above a $\mathrm{pH}$ of around 6.35, indicating the enhancement of buffering capacity of medium was helpful to maintain the $\mathrm{pH}$ stability.

Some studies have reported that the SCFAs produced in the hindgut seems to be derived from the fermentation of nondietary substrates including crude microbial and mucin fractions, which interfered with the determination of dietary fiber fermentation [37]. Supportably, adding mucins to an in vitro batch fermentation model of the large intestine shifted short-chain fatty acid molar ratios, which the proportion of propionate increased with the mucin added [38]. In fact, mucus, act as an adhesion site for some bacterial species [39,40], provided nutrients or a mucosal environment for bacteria to fermentation. Glycerol, as a cryoprotectant, can also fermented by fecal microbiota [12]. In this study, adding glycerol to inoculum decreased the acetate production and increased butyrate and BCFA production compared to inoculum without glycerol fermentation in normal medium. However, the total production of propionate concentration raised steeply after $24 \mathrm{~h}$ with glycerol added, resulting propionate production had a negative net production at $36 \mathrm{~h}$, which indicated addition of glycerin decreased the accuracy of propionic acid determination. An in vitro fermentation study under difference buffer capacity of medium showed that when $\mathrm{pH}$ of medium was 5 or 5.5 , the propionate had a higher ratio in SCFA composition compared to $\mathrm{pH}$ of medium above 6 [41]. This is in concordance with the results obtained in the present study, which showed that the concentration of propionate production increased with the $\mathrm{pH}$ decreased during fermentation. In fact, propionate can be produced from glycerol during in vitro fermentation, and glycerol also can be converted to propylene glycol [42], which could increase propionate and decrease acetate formation [13]. In ruminant researches, the glycerin added in diet fed to beef cattle also increased production of propionate [14]. Meanwhile, another study indicated the concentration of propionic acid increased in dependence of the added glycerol amount and the longer incubation time [43]. Similarly, goats supplemented with glycerol-enriched yeast culture had significantly higher molar proportion of propionate, and significantly lower ruminal pH compared with the control [44]. Therefore, it is speculated that glycerol 
added in fermentation system may obscure the fermentation characteristic of dietary fiber substrates, thereby changing the fermentation profile of microorganisms.

A stable and optimized $\mathrm{pH}$ should be controlled during in vitro fermentation process to promise the maximal microbial activity, since the metabolic activity of microorganisms usually determined fermentable rate in vitro [16]. Therefore, changes in microbial diversity during fermentation is an important indicator to assess the stability of in vitro fermentation system. In addition, differences in the $\mathrm{pH}$ and SCFA in the present study were by far more influenced by medium and inoculum than the substrate. Therefore, we selected digesta of Barley diet to analyze the bacterial community at each time point. Chao1 and Shannon indexes were higher in anti-frozen inoculum than frozen-inoculum at $12 \mathrm{~h}$, whereas lower at $36 \mathrm{~h}$ under the normal medium fermentation. This is mainly because of the decreased $\mathrm{pH}$ (from 6.7 to 5.2) in fermentation broth, which did not suitable for the growth and reproduction of microorganisms. However, under modified medium fermentation, due to the $\mathrm{pH}$ was maintained above 6.3 in anti-frozen inoculum, Chao1 index and Shannon index were similar to that in frozen inoculum at $36 \mathrm{~h}$, indicating modified medium was more suitable for microorganism growth by providing a stable $\mathrm{pH}$ environment. The result was consistent with the previous report that in vitro fermentation of wheat bran (WB) and oat bran (OB) had a lower $\mathrm{pH}$ of fermenters with a lower microbial diversity [16]. Similarly, the results of Bai et al. [11] also showed that the $\alpha$ - diversity (Chao1 and Shannon) were decreased with the decrement of $\mathrm{pH}$ throughout the fermentation process. The $\beta$-diversity of the microbiota in present study showed clear segregations on the microbial communities among different treatments, indicating that the fermentation in different inoculum and medium treatments influenced the microbial structures. The fermentation of inoculum added glycerol and medium with high buffer capacity had a great impact on the microbiota at the phylum and genus level.

Ramayo-Caldas et al. [45] have reported that the dominant microbial phyla of pig feces are Firmicutes and Bacteroidetes, followed by Proteobacteria and Actinobacteria, which is in agreement with in vitro studies [46-48] and also with the current study. The same findings are also observed at genus level that reported by different researchers [11,49], but the abundances are highly variable due to different fermentation substrates or fermentation system. Glycerol usually added to inoculum in study of fecal microbiota transplantation to improve the fecal microbial survival rates during the cryopreservation [50,51]. Our results showed that adding glycerol to inoculum improved Actinoateriota abundance, decreased the abundance of Proteobacteria at $12 \mathrm{~h}$. The modified medium fermentation with frozen inoculum improved Firmcutes and Actinobacteriota at $24 \mathrm{~h}$ and/or $36 \mathrm{~h}$, which were similar to anti-frozen inoculum, and inhabited Proteobacteria growth compared with normal medium. The results indicated that glycerol has a distinct bacteria protection effects, but with the progress of fermentation, the decrement of $\mathrm{pH}$ caused by glycerol fermentation lead to microbial community disorder. However, modified medium would improve the microflora structure. In addition, a recent study showed that $10 \%$ glycerol supplement showed protective effect of fecal microbiota, in which $41.5 \%$ living bacteria in fresh fecal suspension remained alive after frozen with glycerol, rather only $7.1 \%$ bacteria remained alive after direct frozen [52]. Meanwhile, the microbiota composition in glycerol added inoculum was also similar to that of fresh fecal suspension. In the current study, $\alpha$-diversity (Chao1 and Shannon index) of fermentation broth was higher in anti-frozen inoculum at $12 \mathrm{~h}$ of fermentation, as well as high relative abundance of Firmicutes, such as streptococcus, blautia, etc., was observed in anti-frozen inoculum at $12 \mathrm{~h}$ of fermentation. The lower $\mathrm{pH}$ condition would alter the composition and metabolic activity of microflora and therefore changed SCFA profiles. An in vitro study showed that the microbial community composition of rumen fluid at different initial $\mathrm{pH}$ has a significant difference, fermentation used rumen fluid at initial $\mathrm{pH}$ of 6.5 had the maximum utilization rate of fiber, and the rate of fiber degradation declined with the decrease of initial $\mathrm{pH}$ [53], indicating that low $\mathrm{pH}$ suppresses the growth of cellulolytic bacteria. In this research, alterations in the structure 
of the dominant microbiota after $\mathrm{pH}$ decrement could be a reason for a large amount propionate production.

\section{Conclusions}

In this study, adding glycerol to inoculum would decrease $\mathrm{pH}$ of broth, thereby changing the SCFA profiles, especially increased propionate production that elicited large variation of net concentration of propionate and decreased diversity of microbiota. The modified medium could maintain a $\mathrm{pH}$ above 6.3 and maintain the microbial diversity under anti-frozen inoculum. Additionally, modified medium improved the abundance of Firmicutes and Actinobacteria compared with normal medium under frozen inoculum fermentation, indicating modified medium offered a better environment to the fecal microflora. Our results indicated that fermentation using frozen inoculum with modified medium could maintain stability of $\mathrm{pH}$ and improve the accuracy of SCFA determination. However, due to lack of in vitro foregut digestion or have a large difference in the digestion degree compare to in vivo, fermentation characteristics of fiber ingredients evaluated by in vitro fermentation were usually inconsistent with in vivo. In this regard, it might be interesting to include a comparison of the ileal digesta composition with the residues after in vitro simulation of gastrointestinal digestion to improve their similarity in future investigations. Additionally, interindividual variability of fecal microbiota and its contribution to the variability of fermentation need further studied.

Author Contributions: Conceptualization, Q.G., K.L. and L.C.; Date curation, Q.G., L.L. and H.Z. formal analysis, Q.G. and K.L. Investigation, Q.G., K.L. and L.L. Methodology, Q.G., K.L., L.C. and R.Z.; Software, Q.G. and R.Z.; Validation, Q.G., R.Z., L.C. and H.Z.; Writing-Original Draft, Q.G.; Writing-Review and Editing, R.Z., C.L. and Q.G.; Funding acquisition, H.Z. Project administration, L.C. and H.Z. Resource, H.Z. All authors have read and agreed to the published version of the manuscript.

Funding: This study was supported by the Agricultural Science and Technology Innovation Program (CAASZDRW202006-02, ASTIPIAS07) and Agricultural Research Outstanding Talents and In-novation Team (2016-nybrc-03).

Institutional Review Board Statement: Not applicable.

Informed Consent Statement: Not applicable.

Data Availability Statement: The sequence data reported in this study were submitted to the NCBI database (PRJNA793379). The other data presented in this study are available on request from the corresponding author.

Conflicts of Interest: The authors declare no conflict of interest.

\section{References}

1. de Leeuw, J.A.; Bolhuis, J.E.; Bosch, G.; Gerrits, W.J. Effects of dietary fibre on behaviour and satiety in pigs. Proc. Nutr. Soc. 2008, 67, 334-342. [CrossRef]

2. Tan, C.; Wei, H.; Zhao, X.; Xu, C.; Zhou, Y.; Peng, J. Soluble Fiber with High Water-Binding Capacity, Swelling Capacity, and Fermentability Reduces Food Intake by Promoting Satiety Rather Than Satiation in Rats. Nutrients 2016, 8, 615. [CrossRef] [PubMed]

3. Wu, W.; Xie, J.; Zhang, H. Dietary fibers influence the intestinal SCFAs and plasma metabolites profiling in growing pigs. Food Funct. 2016, 7, 4644-4654. [CrossRef]

4. Seo, Y.S.; Lee, H.-B.; Kim, Y.; Park, H.-Y. Dietary Carbohydrate Constituents Related to Gut Dysbiosis and Health. Microorganisms 2020, 8, 427. [CrossRef]

5. Gill, S.K.; Rossi, M.; Bajka, B.; Whelan, K. Dietary fibre in gastrointestinal health and disease. Nat. Rev. Gastroenterol. Hepatol. 2021, 18, 101-116. [CrossRef]

6. Lynd, L.R.; Weimer, P.J.; van Zyl, W.H.; Pretorius, I.S. Microbial cellulose utilization: Fundamentals and biotechnology. Microbiol. Mol. Biol. Rev. 2002, 66, 506-577. [CrossRef]

7. Bayer, E.A.; Belaich, J.P.; Shoham, Y.; Lamed, R. The cellulosomes: Multienzyme machines for degradation of plant cell wall polysaccharides. Annu. Rev. Microbiol. 2004, 58, 521-554. [CrossRef] [PubMed] 
8. Zhao, J.; Bai, Y.; Tao, S.; Zhang, G.; Wang, J.; Liu, L.; Zhang, S. Fiber-rich foods affected gut bacterial community and short-chain fatty acids production in pig model. J. Funct. Foods 2019, 57, 266-274. [CrossRef]

9. Rymer, C.; Huntington, J.; Williams, B.; Givens, D. In vitro cumulative gas production techniques: History, methodological considerations and challenges. Anim. Feed Sci. Technol. 2005, 123, 9-30. [CrossRef]

10. Wang, Y.M. Effects and Mechanisms of Dietary Crude Protein Level on Intestinal Nutrient Digestion and Metabolism in Pigs. Ph.D. Thesis, China Agriculture University, Beijing, China, 2020.

11. Bai, Y.; Zhou, X.; Li, N.; Zhao, J.; Ye, H.; Zhang, S.; Yang, H.; Pi, Y.; Tao, S.; Han, D.; et al. In vitro fermentation characteristics and fiber-degrading enzyme kinetics of cellulose, arabinoxylan, $\beta$-glucan and glucomannan by pig fecal microbiota. Microorganisms 2021, 9, 1071. [CrossRef]

12. Chen, Y.; Wang, T.; Shen, N.; Zhang, F.; Zeng, R.J. High-purity propionate production from glycerol in mixed culture fermentation. Bioresour. Technol. 2016, 219, 659-667. [CrossRef] [PubMed]

13. Trabue, S.; Scoggin, K.; Tjandrakusuma, S.; Rasmussen, M.A.; Reilly, P.J. Ruminal fermentation of propylene glycol and glycerol. J. Agric. Food Chem. 2007, 55, 7043-7051. [CrossRef] [PubMed]

14. Van Cleef, E.H.C.B.; Uwituze, S.; Alvarado-Gilis, C.A.; Miller, K.A.; Van Bibber-Krueger, C.L.; Aperce, C.C.; Drouillard, J.S. Elevated concentrations of crude glycerin in diets for beef cattle: Feedlot performance, carcass traits, and ruminal metabolism1. J. Anim. Sci. 2019, 97, 4341-4348. [CrossRef] [PubMed]

15. Menke, K.H.; Steingass, H. Estimation of the energetic feed value obtained from chemical analysis and in vitro gas production using rumen fluid. Anim. Res. Dev. 1988, 28, 7-55.

16. Bai, Y.; Zhao, J.B.; Tao, S.Y.; Zhou, X.J.; Pi, Y.; Gerrits, W.J.; Johnston, L.J.; Zhang, S.Y.; Yang, H.J.; Liu, L.; et al. Effect of dietary fiber fermentation on short-chain fatty acid production and microbial composition in vitro. J. Sci. Food Agric. 2020, 100, $4282-4291$. [CrossRef]

17. Yu, J.; Fu, Y.; Deng, Z.; Fan, Y.; Li, H. Effects of soluble dietary fiber from soybean residue fermented by Neurospora crassa on the intestinal flora in rats. Food Funct. 2020, 11, 7433-7445. [CrossRef]

18. Wong, J.M.; de Souza, R.; Kendall, C.W.; Emam, A.; Jenkins, D.J. Colonic health: Fermentation and short chain fatty acids. J. Clin Gastroenterol. 2006, 40, 235-243. [CrossRef]

19. Jha, R.; Rossnagel, B.; Pieper, R.; Van Kessel, A.; Leterme, P. Barley and oat cultivars with diverse carbohydrate composition alter ileal and total tract nutrient digestibility and fermentation metabolites in weaned piglets. Animal 2010, 4, 724-731. [CrossRef]

20. Tan, Z.K. Study on the Correlation between Roughage-Resistance and Intestinal Microecological Factors of Tibetan Pigs. Ph.D. Thesis, Tibet University, Xizang, China, 2021.

21. Louis, P.; Flint, H.J. Formation of propionate and butyrate by the human colonic microbiota. Environ. Microbiol. 2017, 19, 29-41. [CrossRef]

22. Zeng, H.; Umar, S.; Rust, B.; Lazarova, D.; Bordonaro, M. Secondary Bile Acids and Short Chain Fatty Acids in the Colon: A Focus on Colonic Microbiome, Cell Proliferation, Inflammation, and Cancer. Int. J. Mol. Sci. 2019, 20, 1214. [CrossRef]

23. Marsono, Y.; Illman, R.J.; Clarke, J.M.; Trimble, R.P.; Topping, D.L. Plasma lipids and large bowel volatile fatty acids in pigs fed on white rice, brown rice and rice bran. Br. J. Nutr. 1993, 70, 503-513. [CrossRef] [PubMed]

24. Uerlings, J.; Schroyen, M.; Bautil, A.; Courtin, C.; Richel, A.; Sureda, E.A.; Bruggeman, G.; Tanghe, S.; Willems, E.; Bindelle, J.; et al. In vitro prebiotic potential of agricultural by-products on intestinal fermentation, gut barrier and inflammatory status of piglets. Br. J. Nutr. 2020, 123, 293-307. [CrossRef]

25. Bindelle, J.; Buldgen, A.; Boudry, C.; Leterme, P. Effect of inoculum and pepsin-pancreatin hydrolysis on fibre fermentation measured by the gas production technique in pigs. Anim. Feed Sci. Technol. 2007, 132, 111-122. [CrossRef]

26. Jonathan, M.C.; van den Borne, J.J.; van Wiechen, P.; da Silva, C.S.; Schols, H.A.; Gruppen, H. In vitro fermentation of 12 dietary fibres by faecal inoculum from pigs and humans. Food Chem. 2012, 133, 889-897. [CrossRef]

27. Liu, Y.; Li, Y.; Ke, Y.; Li, C.; Zhang, Z.; Wu, Y.; Hu, B.; Liu, A.; Luo, Q.; Wu, W. In vitro saliva-gastrointestinal digestion and fecal fermentation of Oudemansiella radicata polysaccharides reveal its digestion profile and effect on the modulation of the gut microbiota. Carbohydr. Polym. 2021, 251, 117041. [CrossRef] [PubMed]

28. Wang, Y. Study on Determination of Protein Availability in Cereal Grains Based on Simulating Gastro-Small Intestinal Digestion Process for Growing Pigs. Master's Thesis, Chinese Academy of Agricultural Sciences, Beijing, China, 2019.

29. Gao, Q.T.; Zhao, F.; Zhang, H.; Wang, Y.; Liu, B.L. Effect of dietary fiber levels and feeding time on passage rate of digesta in the intestinal tract of growing pigs. Chin. J. Anim. Nutr. 2018, 30, 469-476.

30. AOAC. Official Methods of Analysis, 18th ed.; Association of Official Analytical Chemists: Arlington, TX, USA, 2007.

31. Van Soest, P.J.; Robertson, J.B.; Lewis, B.A. Methods for dietary fiber, neutral detergent fiber, and nonstarch polysaccharides in relation to animal nutrition. J. Dairy Sci. 1991, 74, 3583-3597. [CrossRef]

32. Wu, W.; Zhang, L.; Xia, B.; Tang, S.; Xie, J.; Zhang, H. Modulation of Pectin on Mucosal Innate Immune Function in Pigs Mediated by Gut Microbiota. Microorganisms 2020, 8, 535. [CrossRef]

33. Edgar, R.C. UPARSE: Highly accurate OTU sequences from microbial amplicon reads. Nat. Methods 2013, 10, 996-998. [CrossRef]

34. Wang, Q.; Garrity, G.M.; Tiedje, J.M.; Cole, J.R. Naïve Bayesian Classifier for Rapid Assignment of rRNA Sequences into the New Bacterial Taxonomy. App.l Environ. Microbiol. 2007, 73, 5261-5267. [CrossRef]

35. Knudsen, K.B.; Jensen, B.B.; Andersen, J.; Hansen, I. Gastrointestinal implications in pigs of wheat and oat fractions: 2. Microbial activity in the gastrointestinal tract. Br. J. Nutr. 1991, 65, 233-248. [CrossRef] 
36. Collins, S.M.; Gibson, G.R.; Kennedy, O.B.; Walton, G.; Rowland, I.; Commane, D.M. Development of a prebiotic blend to influence in vitro fermentation effects, with a focus on propionate, in the gut. FEMS Microbiol. Ecol. 2021, 97, 101-111. [CrossRef]

37. Montoya, C.A.; Rutherfurd, S.M.; Moughan, P.J. Ileal Digesta Nondietary Substrates from Cannulated Pigs Are Major Contributors to In Vitro Human Hindgut Short-Chain Fatty Acid Production. J. Nutr. 2017, 147, 264-271. [CrossRef] [PubMed]

38. Tran, T.H.T.; Boudry, C.; Everaert, N.; Théwis, A.; Portetelle, D.; Daube, G.; Nezer, C.; Taminiau, B.; Bindelle, J. Adding mucins to an in vitro batch fermentation model of the large intestine induces changes in microbial population isolated from porcine feces depending on the substrate. FEMS Microbiol. Ecol. 2016, 92, fiv165. [CrossRef] [PubMed]

39. Macfarlane, S.; Woodmansey, E.J.; Macfarlane, G.T. Colonization of mucin by human intestinal bacteria and establishment of biofilm communities in a two-stage continuous culture system. Appl. Environ. Microbiol. 2005, 71, 7483-7492. [CrossRef]

40. Van Tassell, M.L.; Miller, M.J. Lactobacillus adhesion to mucus. Nutrients 2011, 3, 613-636. [CrossRef] [PubMed]

41. Lu, C.; Tahir, N.; Li, W.; Zhang, Z.; Jiang, D.; Guo, S.; Wang, J.; Wang, K.; Zhang, Q. Enhanced buffer capacity of fermentation broth and biohydrogen production from corn stalk with NaHPO/NaHPO. Bioresour. Technol. 2020, 313, 783-788. [CrossRef]

42. Mu, Y.; Teng, H.; Zhang, D.-J.; Wang, W.; Xiu, Z.L. Microbial production of 1,3-propanediol by Klebsiella pneumoniae using crude glycerol from biodiesel preparations. Biotechnol. Lett. 2006, 28, 1755-1759. [CrossRef]

43. Bergner, H.; Kijora, C.; Ceresnakova, Z.; Szakacs, J. In vitro studies on glycerol transformation by rumen microorganisms. Arch. Tierernahr. 1995, 48, 245-256. [CrossRef]

44. Ye, G.; Zhu, Y.; Liu, J.; Chen, X.; Huang, K. Preparation of glycerol-enriched yeast culture and its effect on blood metabolites and ruminal fermentation in goats. PLoS ONE 2014, 9, 410-418. [CrossRef]

45. Ramayo-Caldas, Y.; Mach, N.; Lepage, P.; Levenez, F.; Denis, C.; Lemonnier, G.; Leplat, J.-J.; Billon, Y.; Berri, M.; Doré, J.; et al Phylogenetic network analysis applied to pig gut microbiota identifies an ecosystem structure linked with growth traits. ISME J. 2016, 10, 2973-2977. [CrossRef]

46. Wang, M.; Chen, G.; Chen, D.; Ye, H.; Sun, Y.; Zeng, X.; Liu, Z. Purified fraction of polysaccharides from Fuzhuan brick tea modulates the composition and metabolism of gut microbiota in anaerobic fermentation in vitro. Int. J. Biol. Macromol. 2019, 140, 858-870. [CrossRef]

47. Rui, Y.; Wan, P.; Chen, G.; Xie, M.; Sun, Y.; Zeng, X.; Liu, Z. Simulated digestion and fermentation in vitro by human gut microbiota of intra- and extra-cellular polysaccharides from Aspergillus cristatus. LWT-Food Sci. Technol. 2019, 116, 108508. [CrossRef]

48. Long, C.; de Vries, S.; Venema, K. Differently Pre-treated Rapeseed Meals Affect in vitro Swine Gut Microbiota Composition. Front. Microbiol. 2020, 11, 570985. [CrossRef]

49. Han, K.H.; Enomoto, M.; Pelpolage, S.; Nagata, R.; Fukuma, N.; Fukushima, M. In vitro fermentation potential of the residue of Korean red ginseng root in a mixed culture of swine faecal bacteria. Food Funct. 2020, 11, 6202-6214. [CrossRef] [PubMed]

50. Lee, C.H.; Steiner, T.; Petrof, E.O.; Smieja, M.; Roscoe, D.; Nematallah, A.; Weese, J.S.; Collins, S.; Moayyedi, P.; Crowther, M.; et al Frozen vs Fresh Fecal Microbiota Transplantation and Clinical Resolution of Diarrhea in Patients With Recurrent Clostridium difficile Infection: A Randomized Clinical Trial. JAMA 2016, 315, 142-149. [CrossRef]

51. Hu, J.; Chen, L.; Tang, Y.; Xie, C.; Xu, B.; Shi, M.; Zheng, W.; Zhou, S.; Wang, X.; Liu, L.; et al. Standardized Preparation for Fecal Microbiota Transplantation in Pigs. Front. Microbiol. 2018, 9, 1328-1337. [CrossRef] [PubMed]

52. Fu, X.C.; Chen, A.L.; Zhang, Y.J.; Pang, X.Y. Protective effect of cryoprotectant on viable bacteria in fecal suspension. Genom. Appl. Biol. 2020, 39, 5586-5592.

53. Zhang, L.; Xing, D.F.; Jiang, Q.Q.; Sun, R.; Ren, N.Q. Properties of cellulose degrading hydrogen producing bacteria flora in rumen fluid at different pH. J. Dalian Polytech. Univ. 2017, 36, 328-332. 